\title{
SERS spectra of a single nasopharyngeal carcinoma cell based on intracellularly grown and passive uptake Au nanoparticles
}

\author{
Hao Huang a, Weiwei Chen ${ }^{\text {a,b }}$, Jianji Pan ${ }^{c}$, Qisong Chen ${ }^{c}$, Shangyuan Feng ${ }^{b, *}$, Yun Yu ${ }^{\text {a,b }}$, \\ Yanping Chen ${ }^{\mathrm{d}}$, Ying $\mathrm{Su}^{\mathrm{c}}$ and Rong Chen ${ }^{\mathrm{b}}$ \\ ${ }^{\text {a }}$ College of Integrated Traditional Chinese and Western Medicine, Fujian University of Traditional \\ Chinese Medicine, Fuzhou, China \\ ${ }^{\mathrm{b}}$ Key Laboratory of Optoelectronic Science and Technology for Medicine, Ministry of Education, \\ Fujian Provincial Key Laboratory of Photonic Technology, Fujian Normal University, Fuzhou, China \\ ${ }^{\mathrm{c}}$ Fujian Provincial Cancer Hospital, Fuzhou, China \\ ${ }^{\mathrm{d}}$ Fujian Provincial Tumor Hospital, Fuzhou, China
}

\begin{abstract}
The intracellularly-grown-Au-nanoparticles (IGAuNs) technique was employed to analyze the surface-enhanced Raman scattering (SERS) spectra of nasopharyngeal carcinoma cells (CNE-1 cell line). There are only six obvious Raman bands $\left(718,1001,1123,1336,1446,1660 \mathrm{~cm}^{-1}\right)$ in the normal Raman spectrum of living CNE-1 cells. However, over twenty SERS Raman bands have been detected in the SERS spectra of IGAuNs-induced cells, among which five bands are of the DNA backbone $\left(673,1097,1306,1336\right.$ and $\left.1585 \mathrm{~cm}^{-1}\right)$. There are four vibrations of the DNA backbone $(1026,1097,1336$ and $1585 \mathrm{~cm}^{-1}$ ) in the SERS spectra of living CNE-1 cells induced by the passive uptake gold nanoparticles (PUAuNS), but one more DNA backbone and many nucleus Raman peaks appeared in the IGAuNs-induced SERS spectra. Many Raman peaks in the PUAuNs-induced SERS spectra are stronger than those in the IGAuNs-induced ones. This study has shown that the PUAuNs technique can achieve stronger Raman signals, and that the IGAuNs technique can enable the gold element to access to the nucleus more easily, which could help to obtain more surface-enhanced Raman signals of the intracellular biochemical molecules. Thus, the two techniques can work together to attain the Raman spectral information of the cytoplasm and the nucleus in a better way, which might provide a sensitive method for broad biomedical applications such as intracellular SERS analysis of living cells.
\end{abstract}

Keywords: Surface-enhanced Raman scattering (SERS), intracellularly grown gold nanoparticles (IGAuNs), passively uptaken gold nanoparticles (PUAuNS), nasopharyngeal carcinoma cell

\section{Introduction}

Surface-enhanced Raman scattering (SERS) has demonstrated great potential for probing living cells, because it can increase the Raman cross section by $10^{10}-10^{14}$ orders of magnitude $[8,18]$. In current intracellular SERS measurements, nanoparticles are delivered into living cells by passive uptake [10]. A transfer of silver or gold nanoparticles into cell culture medium should result in nanoparticles coated

\footnotetext{
*Corresponding author: Dr. Shangyuan Feng, Key Laboratory of Optoelectronic Science and Technology for Medicine, Ministry of Education, Fujian Provincial Key Laboratory for Photonics Technology, Fujian Normal University, Fuzhou 350007, China. Tel.: +86 591 83489919; Fax: +86 591 83465373; E-mail: syfeng@ @jnu.edu.cn.
} 
with proteins like BSA (bovine serum albumin) that adsorb to the nanoparticle surface, and after periods of time, this coating should be internalization by living cells through phagocytosis and/or endocytosis [7]. And then, the living cells were washed and prepared for biological assays, electron microscopy, and Raman measurements. The SERS spectra of a single living human nasopharyngeal carcinoma induced by passively uptaken gold nanoparticles (PUAuNS) have been obtained in our previous study [6]. However, introducing nanoparticles into the cells is dependent on the cell's "passive uptake", which makes it difficult to deliver a nanometer sized colloidal metal particle into nucleolus $[3,16]$.

In recent years, it have been demonstrated that different microorganisms, including yeast and actinomycetes, can reduce metallic ions into elemental metals and synthesize nanoparticles of different shapes, sizes and compositions $[13,14]$. The intracellularly-grown-Au-nanoparticles (IGAuNs) technique is that cells are incubated with gold ions, which will enter the cytoplasm and the nuclear and then be reduced into gold nanoparticles [13]. Since the IGAuNs technique provides superior delivery efficiency to cytoplasm and nucleus for the elemental gold, more Raman signals of native chemicals within a cell could be detected. There are many methods for diagnosing nasopharyngeal carcinoma cells (NPC cells), such as irradiation-inducing, immunochemistry, gene research and photodynamic therapy. Nevertheless, there still exist some difficulties in the early diagnosis of nasopharyngeal carcinoma. Hence, a sensitive and structurally selective detecting method should be found, and the SERS has shown promise in detecting the NPC cells by using metal nanoprobes. After a series of experiments, our team has gained some meaningful results $[4,5,11,12,21]$.

The IGAuNs technique has been employed to detect MCF10 epithelial cells in the report of Shamsaie et al. [16]. In this study, we used IGAuNs technique and NIR laser excitation for the SERS analysis of the intracellular biochemical composition for the first time. Moreover, we compared the SERS spectra between PUAuNs-induced and IGAuNs-induced. Human nasopharyngeal carcinoma cell line (CNE-1) was used as a model in our study.

\section{Experimental methods}

Cell culture. Human nasopharyngeal carcinoma cells (CNE-1 cell line) were obtained from the Fujian Provincial Tumor Hospital and were cultured by Fujian normal university. The CNE-1 cells were grown as monolayers in RPMI 1640 medium (supplemented with $100 \mathrm{IU} / \mathrm{ml}$ penicillin/streptomycin and 10\% fetal calf serum, hereafter referred to as complete RPMI 1640) at $37^{\circ} \mathrm{C}$ and $5 \% \mathrm{CO}_{2}$ with $100 \%$ relative humidity.

Intracellularly grown gold nanoparticles. CNE-1 cells were harvested by removing the medium, rinsing the cells with $0.25 \%$ of trypsin in a phosphate-buffer ( $\mathrm{pH}$ 7.4) containing $1 \mathrm{mM}$ of EDTA and $25 \mathrm{mM}$ of HEPES. After incubation at $37^{\circ} \mathrm{C}$ for $10 \mathrm{~min}$, the action of the trypsin was stopped by adding $2 \mathrm{ml}$ complete RPMI-1640 medium, and the cells were diluted to a concentration of $10^{5}$ cells $/ \mathrm{ml}$. The resuspended cells ( $4 \mathrm{ml}$ ) were placed in a $35 \mathrm{~mm}$ Petri dish containing a thin quartz glass (thickness: $1 \mathrm{~mm}$, radius: $7.5 \mathrm{~mm}$ ). Since the CNE-1 cells are an adherent cell line, it can grow on the quartz glass, which is easy for the detection of Raman spectra. When CNE-1 cells grew to $80 \%$ of the quartz glass surface, the medium was moved and replaced with phosphate buffer saline (PBS, pH 7.4) and incubated with $1 \mathrm{mM} \mathrm{HAuCl}{ }_{4}$ solution in culture well. After $36 \mathrm{~h}$, intracellularly grown gold nanoparticles formed, which could be indicated by the appearance of a pink color in Petri dish [16]. We also had CNE-1 cells incubated with the PBS buffer without $\mathrm{HAuCl}_{4}$ for $36 \mathrm{~h}$ as the negative control experiment and no color change was observed in the negative controls. Moreover, solutions of $\mathrm{HAuCl}_{4}$ in $\mathrm{PBS}$ are stable for 


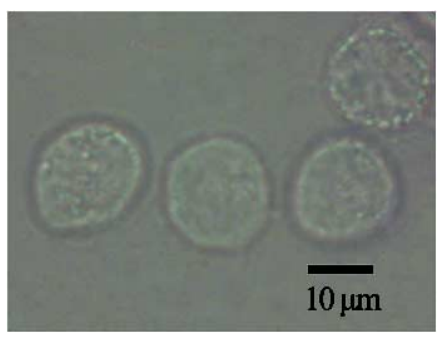

Fig. 1. Optical microscopic image of living CNE-1 cells adhere to the thin quartz glass $(\times 50)$. (Colors are visible in the online version of the article; http://dx.doi.org/10.3233/SPE-2011-0540.)

months, so the possibility of autoreduction is ruled out. Figure 1 shows the cell morphology of CNE-1 cells which adhere to the thin quartz glass. The CNE-1 cell is elliptic and about $18 \mu \mathrm{m}$. Before the Raman measurements, the medium was removed and two groups of cells including negative control and test (cells incubated with IGAuNs) were washed three times with PBS to ensure any medium loosely attached to the cell membrane were washed out. And then, Raman measurements are carried out while the cells are alive in the PBS buffer.

Preparation of gold nanoparticles. Stable gold colloids were prepared according to the method reported by Grabar et al. Briefly, $50 \mathrm{ml}$ of $\mathrm{HAuCL}_{4}\left(10^{-4} \mathrm{~g} / \mathrm{ml}\right)$ was brought to a rolling boil with vigorous stirring. Rapid addition of $5 \mathrm{ml}$ of $1 \%$ sodium citrate to the vortex of the solution resulted in a color change from pale yellow to burgundy. Boiling and stirring was continued for an additional $15 \mathrm{~min}$ and then the solution was cooled naturally.

Passive uptake Au nanoparticles. $4 \mathrm{ml} \mathrm{CNE}-1$ cells suspension (concentration of $10^{5}$ cells $/ \mathrm{ml}$ ) were placed in a $35 \mathrm{~mm}$ Petri dish containing a thin quartz glass (thickness: $1 \mathrm{~mm}$, radius: $7.5 \mathrm{~mm}$ ). The cells were allowed to settle (adhere) in a $\mathrm{CO}_{2}$ incubator at $37^{\circ} \mathrm{C}$. Once the $\mathrm{CNE}-1$ cells grew to $80 \%$ of the quartz glass surface, $1 \mathrm{ml}$ gold colloids were added to the cell culture medium and the cells were allowed to grow for $36 \mathrm{~h}$ and were then harvested followed by thorough washing of the cells before Raman measurements.

Raman measurement. A Renishaw Raman microscope (InVia System) with a $\sim 2 \lambda$ spatial resolution, a $20 \mathrm{~mW}, 785 \mathrm{~nm}$, semiconductor laser as excitation source, was used for the collection of SERS spectra. The microscope was operated under a $50 \times$ objective, which focused the laser beam onto a spot on the cell and the illumination pinhole was adjusted for the laser spot to cover the whole cell. The typical spectral accumulation time in this study was $10 \mathrm{~s}$ and Raman spectra were scanned over a wavenumber range of 450-2000 $\mathrm{cm}^{-1}$. Peak frequency calibration and rapid checking of instrumental performance were performed with the silicon phonon line at $520 \mathrm{~cm}^{-1}$. An automated algorithm for autofluorescence background removal was applied to the measured raw data to extract pure Raman spectra. The program was kindly offered by the BC Cancer Research Centre [23].

\section{Result and discussion}

\subsection{SERS spectra induced by IGAuNs and NRS from a single living CNE-1 cell}

Figure 2 shows the SERS spectra collected from a single living IGAuNs-treated CNE-1 cell (spectrum (a)) and the normal Raman spectrum (NRS) from the negative control (spectrum (b)), respectively. 


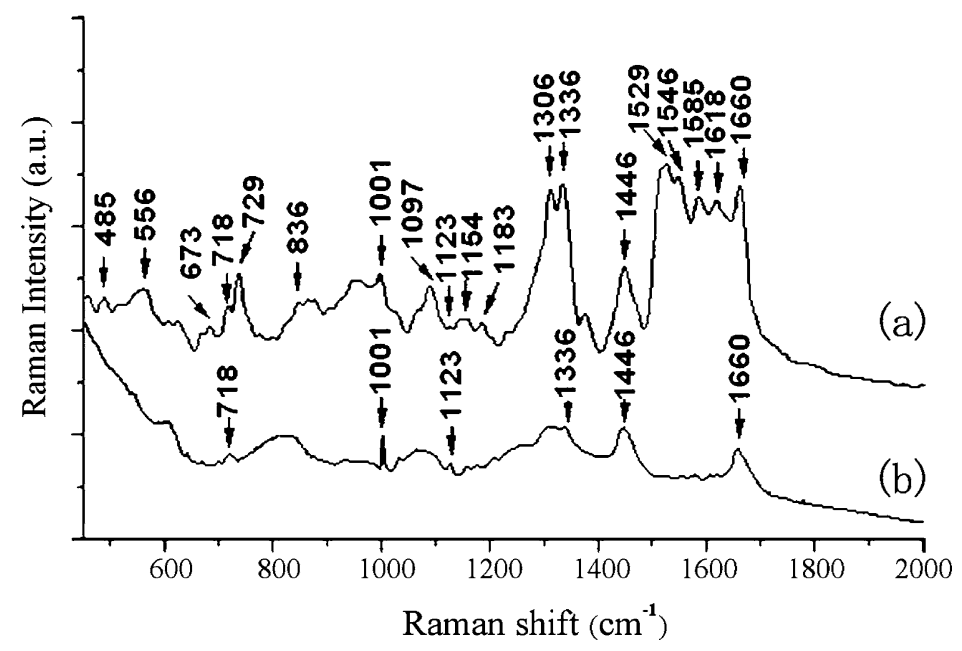

Fig. 2. SERS spectrum (a) induced by IGAuNs and NRS (b) from single living CNE-1 cells.

Spectrum (a) shows example SERS spectrum measured from individual living CNE-1 cells induced by IGAuNs. High quality SERS spectra are obtained, containing a wealth of intracellular SERS signals. Many bands in this spectrum can be assigned to cytoplasmic composition, such as $836 \mathrm{~cm}^{-1}$ (tyrosine), $1123 \mathrm{~cm}^{-1}$ (lipids: $\mathrm{C}-\mathrm{C}$ stretch/protein: $\mathrm{C}-\mathrm{N}$ stretch), $1446 \mathrm{~cm}^{-1}$ (protein: $\mathrm{C}-\mathrm{H}_{2}$ deformation), $1618 \mathrm{~cm}^{-1}$ (tryptophan), etc. The SERS spectrum contains information about nucleus as expected: the SERS band at $673,1097,1306,1336$ and $1585 \mathrm{~cm}^{-1}$ which are contributed by the DNA backbone; the band at $556 \mathrm{~cm}^{-1}$ which is assigned to uracil; the peak $1154 \mathrm{~cm}^{-1}$ which is related to deoxyribose; the band at $1546 \mathrm{~cm}^{-1}$ which is related to guanine; etc. Five bands of DNA backbone demonstrated that the intracellularly grown gold nanoparticles were able to enter the nucleus and formed the SERSactive clusters. Table 1 lists tentative assignments for the observed SERS bands, according to some literature data $[1,2,9,15,22]$.

As shown in spectrum (b), six Raman bands $\left(718,1001,1123,1336,1446,1660 \mathrm{~cm}^{-1}\right)$ were observed in the NRS of living CNE-1 cells for negative control (incubated with the PBS buffer without $\mathrm{HAuCl}_{4}$ ). However, owing to the intracellularly grown gold nanoparticles that have entered cells, the intensity of Raman bands (1336, 1446 and $1660 \mathrm{~cm}^{-1}$ ) of NRS has significantly enhanced in the SERS spectra. Many Raman bands in SERS spectra induced by IGAuNs did not appear in the NRS, which indicated that IGAuNs could sense the native chemicals inside a cell.

Compared with the NRS (spectrum (b)), the Raman band at $1001 \mathrm{~cm}^{-1}$, which is assigned to the symmetric ring breathing mode of phenylalanine, becomes significantly weaker in the SERS spectra induced by IGAuNs (spectrum (a)), which might be related to the changes in the living environment of CNE-1 cells. Compared with the negative control experiment (CNE-1 cells grew in the PBS buffer without $\mathrm{HAuCl}_{4}$ for $36 \mathrm{~h}$ ), CNE-1 cells were incubated with $1 \mathrm{mM} \mathrm{HAuCl}_{4}$ solution in the PBS in order to form intracellular grown gold nanoparticles. However, due to its favorable physical and chemical properties and biocompatibility, gold should be the more suitable metal for incorporation inside living cells, CNE-1 cells had to stand in the hostile environment where they resided. Since the damage of cell viability, the hydrogen bond from the link of phenylalanine broke off and the link structure unfolded, which accounted for the decrease of Raman intensity of the $1001 \mathrm{~cm}^{-1}$ line. According to some report, the cell viability might also affect the intensity of Raman bands $\left(718\right.$ and $\left.1123 \mathrm{~cm}^{-1}\right)$ in the SERS spectra [16]. 
Table 1

SERS peak positions and tentative assignments of major vibrational bands in the SERS spectra induced by IGAuNs

\begin{tabular}{ll}
$\begin{array}{l}\text { Peak positions } \\
\left(\mathrm{cm}^{-1}\right)\end{array}$ & \multicolumn{1}{c}{ Tentative assignment } \\
\hline 485 & The base groups and sugar \\
556 & Uracil \\
673 & DNA backbone \\
718 & Ribose-phosphoric acid \\
729 & Thymine \\
836 & Tyrosine \\
1001 & Symmetric ring breathing mode of phenylalanine \\
1097 & DNA backbone \\
1123 & Lipids: C-C stretch/protein: C-N stretch \\
1154 & Deoxyribose \\
1183 & Non-base group: C-N stretch \\
1306 & DNA/RNA \\
1336 & DNA \\
1446 & Protein: C-H 2 deformation \\
1529 & Cytosine \\
1546 & Guanine \\
1585 & Planar ring of DNA/RNA \\
1618 & Tryptophan \\
1660 & Amide I \\
\hline
\end{tabular}

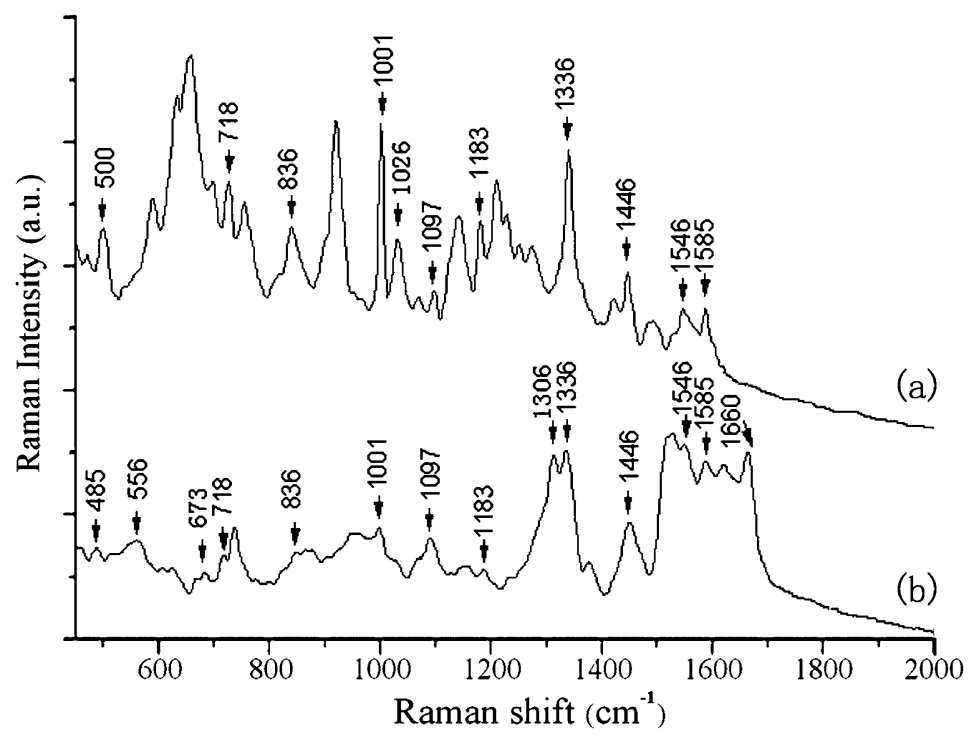

Fig. 3. Comparison between PUAuNs (a) and IGAuNs (b) induced SERS spectra from single living CNE-1 cells.

\subsection{Comparison between PUAuNs-induced and IGAuNs-induced SERS spectra}

In a separate experiment, SERS spectra induced by passive uptake (incubated with gold nanoparticles) were collected and compared with the IGAuNs-induced SERS. As shown in Fig. 3, spectrum (a) 
shows example SERS spectrum measured from individual living CNE-1 cells induced by PUAuNs, and spectrum (b) shows example SERS spectrum measured from individual living CNE-1 cells induced by IGAuNs. Figure 3 demonstrates that many Raman peaks are the same in both spectrum (a) and (b), such as $718,836,1001,1097,1183,1336,1446,1546$ and $1585 \mathrm{~cm}^{-1}$, etc. Therefore, PUAuNs and IGAuNs have similar properties.

As shown in spectrum (a), there are four vibrations of the DNA backbone (1026, 1097, 1336 and $1585 \mathrm{~cm}^{-1}$ ) and other Raman bands related to the nucleus are as follows: the $718 \mathrm{~cm}^{-1}$ peak that is assigned to ribose-phosphoric acid, the bands at 650 and $753 \mathrm{~cm}^{-1}$ that are assigned to guanine and thymine. As shown in spectrum (b), the Raman bands of 673, 1097, 1306, 1336 and $1585 \mathrm{~cm}^{-1}$ are assigned to vibrations of the DNA backbone, and there are many other Raman lines related to the nucleus, for example, $556 \mathrm{~cm}^{-1}$ (uracil), $718 \mathrm{~cm}^{-1}$ (ribose-phosphoric acid), $729 \mathrm{~cm}^{-1}$ (thymine), $1183 \mathrm{~cm}^{-1}$ (non-base group: $\mathrm{C}-\mathrm{N}$ stretch), $1529 \mathrm{~cm}^{-1}$ (cytosine) and $1546 \mathrm{~cm}^{-1}$ (guanine). Therefore, IGAuNsinduced SERS spectrum shows one more DNA backbone and much more nuclear Raman information. In contrast to the PUAuNs technique, the IGAuNs technique can enable the gold ion to access the nucleus more easily. Moreover, the $1660 \mathrm{~cm}^{-1}$ peak in NRS was also observed in the IGAuNs-induced SERS spectrum except for PUAuNs, which indicated that IGAuNs shows a preferable selecting capacity.

Comparing two SERS spectra induced by different technology (PUAuNs-induced and IGAuNsinduced) in Fig. 3, an obvious difference between the PUAuNs- and IGAuNs-induced SERS spectra is that the Raman intensity of the PUAuNs-induced SERS spectrum is much stronger than that of the IGAuNs-induced SERS spectra, such as $718,836,1001,1183 \mathrm{~cm}^{-1}$, etc. There might be two possible reasons accounting for the changes of intensity. On the one hand, the cell viability of CNE-1 cells decreased when the culture media was replaced by the $\mathrm{PBS} / \mathrm{HAuCl}_{4}$ mixture for $36 \mathrm{~h}$, which directly led to the weakening of the Raman signals. On the other hand, the gold nanoparticles used in PUAuNs were easily prepared by chemical reduction, and the formation of gold nanoparticles in IGAuNs was greatly dependent on the intracellular environment. Recent investigations have shown that the aggregated gold nanoparticles clusters generate SERS hot spots where the Raman signals are enhanced a few orders of magnitude [5]. Consequently, gold nanoparticles prepared by chemical reduction used in PUAuNs showed preferable physical properties (such as the size and the shape) and enhancing effect than the intracellularly grown gold nanoparticles. Moreover, the sharp Raman peak around $500 \mathrm{~cm}^{-1}$ which appeared in the PUAuNs-induced SERS spectrum (spectrum (a)) was absent in the IGAuN-induced SERS spectrum (spectrum (b)). This Raman peak is also present in the PUAuNs SERS spectra from previous studies $[6,16]$. It can be related to the stretching vibration mode of disulfide bond $\left(\nu_{\mathrm{S}-\mathrm{S}}\right)$. According to some literature data, this can be indicative of lysosomal proteins that have an abundance of disulfide bonds $[15,16]$. To form the intracellularly grown gold nanoparticles, CNE-2 cells were incubated with $1 \mathrm{mM} \mathrm{HAuCl}_{4}$ solution in the PBS buffer and lysosomal proteins need to resist the hostile conditions of the environment in which they reside. The oxidizing environment of lysosomes as opposed to the reducing environment of the cytosol favors the formation of disulfide bond and eventually resulted in the absence of $500 \mathrm{~cm}^{-1}$ line in the IGAuN-induced SERS spectra.

According to some report, the reproducibility of whole-cell SERS spectra, in fact an integration or average of SERS signals from all micro-locations within the cell, have improved dramatically over the point SERS spectra measured with micron size laser beam [12]. In this study, we focused the laser beam onto a spot on the cell and adjusted the illumination pinhole for the laser spot to cover the whole cell. However, the SERS spectra show slight fluctuations. This is a very interesting feature of SERS called "blinking effect" already reported in literature, but not yet fully understood [17]. Moreover, as a result of tiny movement and biochemical metabolism of living cells during SERS measurement and the 
spectral selectivity and high sensitivity of the SERS, the intensity of the observed Raman bands were varied considerably [19]. In addition, the heating by the laser excitation enhances the surface diffusion of adsorbed cellular molecules, which also leads to the signal fluctuations [20].

\section{Conclusion}

In summary, the IGAuNs-induced SERS spectra of living human nasopharyngeal carcinoma cells (CNE-1 cell line) were reported for the first time in this paper. There are only six obvious Raman bands $\left(718,1001,1123,1336,1446\right.$ and $\left.1660 \mathrm{~cm}^{-1}\right)$ in the normal Raman spectra of living CNE-1 cells from negative control, while over twenty strong SERS Raman bands were observed in the IGAuNsinduced SERS spectra since the IGAuNs have formed inside the cells, where the intensity of Raman bands $\left(1336,1446\right.$ and $1660 \mathrm{~cm}^{-1}$ ) has significantly increased. However, other Raman bands in NRS cannot be observed in IGAuNs-induced SERS spectra, which might be related to the change of the living environment of CNE-1 cells. Four vibrations of the DNA backbone (1026, 1097, 1336 and $1585 \mathrm{~cm}^{-1}$ ) were observed in the PUAuNs-induced SERS spectra, while one more DNA backbone and many more nuclear Raman peaks appeared in the IGAuNs-induced SERS spectra. The intensity of many Raman peaks in the PUAuNs-induced SERS spectra was stronger than that in the IGAuNs-induced ones.

The results show that the PUAuNs technique can detect stronger Raman signals, and the IGAuNs technique can enable the gold nanosensor to access the nucleus more easily, which could help to detect more Raman signals of the biochemical molecules of nucleus. Thus, the PUAuNs technique and the IGAuNs techniques may work together to obtain the Raman spectra of the cytoplasm and the nucleus in a better way, which might offers great potential for biomedical applications such as intracellular SERS analysis of living cells.

\section{Acknowledgements}

The study was supported by the National Natural Science Foundation of China (Nos 11104030, 61178090, 81101110), the Project of Fujian Province (Nos 2008J0016, 2009J01276, JA11055), the Project of Science Foundation of Ministry of Health and United Fujian Provincial Health and Education Project for Tackling the Key Research (No. WKJ2008-2-046), and the Project of State Key Laboratory of Physical Chemistry of Solid Surface and Fujian Key Lab. of Semiconductors and Applications, Xiamen University. We greatly appreciate all the support of the above organizations and the assistance from Dr. Haishan Zeng and Dr. Zhiwei Huang.

\section{References}

[1] J.W. Chan, D.S. Taylor, T. Zwerdling, S.M. Lane, K. Ihara and T. Huser, Micro-Raman spectroscopy detects individual neoplastic and normal hematopoietic cells, Biophysical Journal 90 (2006), 648-656.

[2] Y. Chen, Y.Z. Li, Y. Su, J.Q. Lin, J.J. Pan, R. Chen, C.Y. Zou, S. Lin and C. Li, Raman spectra and discrimination of NPC cell line CNE1 and normal nasopharyngeal cell line NP69, Progress in Biomedical Optics and Imaging 10 (2009), 75191E.1-75191E.7.

[3] B.D. Chithrani, A.A. Ghazani and W.C.W. Chan, Determining the size and shape dependence of gold nanoparticle uptake into mammalian cells, Nano Letters 6 (2006), 662-668.

[4] S. Feng, R. Chen, J. Lin, J. Pan, G. Chen, Y. Li, M. Cheng, Z. Huang, J. Chen and H. Zeng, Nasopharyngeal cancer detection based on blood plasma surface-enhanced Raman spectroscopy and multivariate analysis, Biosensors and Bioelectronics 25 (2010), 2414-2419. 
[5] S. Feng, J. Lin, M. Cheng, Y.Z. Li, G. Chen, Z. Huang, Y. Yu, R. Chen and H. Zeng, Gold nanoparticle based surfaceenhanced Raman scattering spectroscopy of cancerous and normal nasopharyngeal tissues under near-infrared laser excitation, Applied Spectroscopy 63 (2009), 1089-1094.

[6] H. Huang, J.J. Pan, W.W. Chen, Q.S. Chen, S.Y. Feng, Y. Su, X.W. Xu and R. Chen, Surfaced-enhanced Raman spectroscopic study on single living human nasopharyngeal carcinoma cells incubated with colloidal gold, Spectra and Spectral Analysis 11 (2010), 2981-2984.

[7] J. Kneipp, Nanosensors based on SERS for applications in living cells, Surface-Enhanced Raman Scattering 103 (2006), $335-349$.

[8] J. Kneipp, H. Kneipp, A. Rajadurai, R.W. Redmond and K. Kneipp, Optical probing and imaging of live cells using SERS labels, Journal of Raman Spectroscopy 40 (2009), 1-5.

[9] J. Kneipp, H. Kneipp, B. Wittig and K. Kneipp, Novel optical nanosensors for probing and imaging live cells, Nanomedicine 6 (2010), 214-226.

[10] K. Kneipp, A.S. Haka, H. Kneipp, K. Badizadegan, N. Yoshizawa, C. Boone, K.E. Shafer-Peltier, J.T. Motz, R.R. Dasari and M.S. Feld, Surface-enhanced Raman spectroscopy in single living cells using gold nanoparticles, Applied Spectroscopy 56 (2002), 150-154.

[11] D. Lin, J. Lin, Y. Wu, S. Feng, Y. Li, Y. Yu, G. Xi, H. Zeng and R. Chen, Investigation on the interactions of lymphoma cells with paclitaxel by Raman spectroscopy, Spectroscopy 25 (2011), 23-32.

[12] J. Lin, R. Chen, S. Feng, Y. Li, Z. Huang, S. Xie, Y. Yu, M. Cheng and H. Zeng, Rapid delivery of silver nanoparticles into living cells by electroporation for surface-enhanced Raman spectroscopy, Biosensors and Bioelectronics 25 (2009), 388-394.

[13] J.R. Lloyd, Microbial reduction of metals and radionuclides, FEMS Microbiology Reviews 27 (2003), 411-425.

[14] D. Mandal, M. Bolander, D. Mukhopadhyay, G. Sarkar and P. Mukherjee, The use of microorganisms for the formation of metal nanoparticles and their application, Applied Microbiology and Biotechnology 69 (2006), 485-492.

[15] Z. Movasaghi, S. Rehman and I. Rehman, Raman spectroscopy of biological tissues, Applied Spectroscopy Reviews 42 (2007), 493-541.

[16] A. Shamsaie, M. Jonczyk, J. Sturgis, J.P. Robinson and J. Irudayaraj, Intracellularly grown gold nanoparticles as potential surface-enhanced Raman scattering probes, Journal of Biomedical Optics 12 (2007), 020502.

[17] M. Sládková, B. Vlcková, I. Pavel, K. Sisková and M. Slouf, Surface-enhanced Raman scattering from a single molecularly bridged silver nanoparticle aggregate, Journal of Molecular Structure 924-926 (2009), 567-570.

[18] A. Sujith, T. Itoh, H. Abe, K. Yoshida, M.S. Kiran, V. Biju and M. Ishikawa, Imaging the cell wall of living single yeast cells using surface-enhanced Raman spectroscopy, Analytical and Bioanalytical Chemistry 394 (2009), 1803-1809.

[19] A. Taleb, J. Diamond, J.J. McGarvey, J.R. Beattie, C. Toland and P.W. Hamilton, Raman microscopy for the chemometric analysis of tumor cells, Journal of Physical Chemistry B 110 (2006), 19625-19631.

[20] H.W. Tang, X.B. Yang, J. Kirkham and D.A. Smith, Probing intrinsic and extrinsic components in single osteosarcoma cells by near-infrared surface-enhanced Raman scattering, Analytical Chemistry 79 (2007), 3646-3653.

[21] Y. Yu, J. Lin, Y. Wu, S. Feng, Y. Li, Z. Huang, R. Chen and H. Zeng, Optimizing electroporation assisted silver nanoparticle delivery into living C666 cells for surface-enhanced Raman spectroscopy, Spectroscopy - Biomed. Appl. 25 (2011), $13-21$.

[22] E. Zachariah, A. Bankapur, C. Santhosh, M. Valiathan and D. Mathur, Probing oxidative stress in single erythrocytes with Raman tweezers, J. Photochem. Photobiol. B 100 (2010), 113-116.

[23] J. Zhao, H. Lui, D.I. McLean and H. Zeng, Automated autofluorescence background subtraction algorithm for biomedical Raman spectroscopy, Applied Spectroscopy 61 (2007), 1225-1232. 


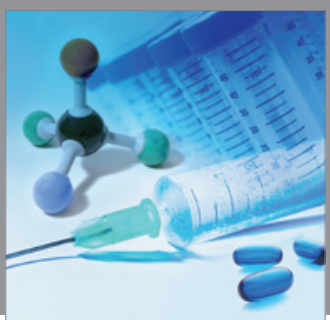

International Journal of

Medicinal Chemistry

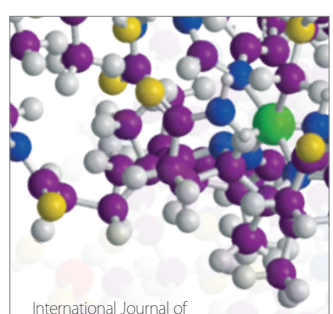

Carbohydrate Chemistry

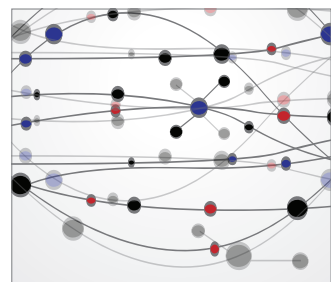

The Scientific World Journal
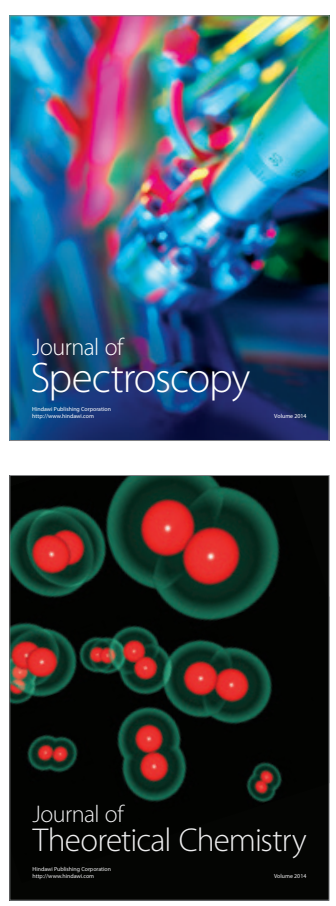
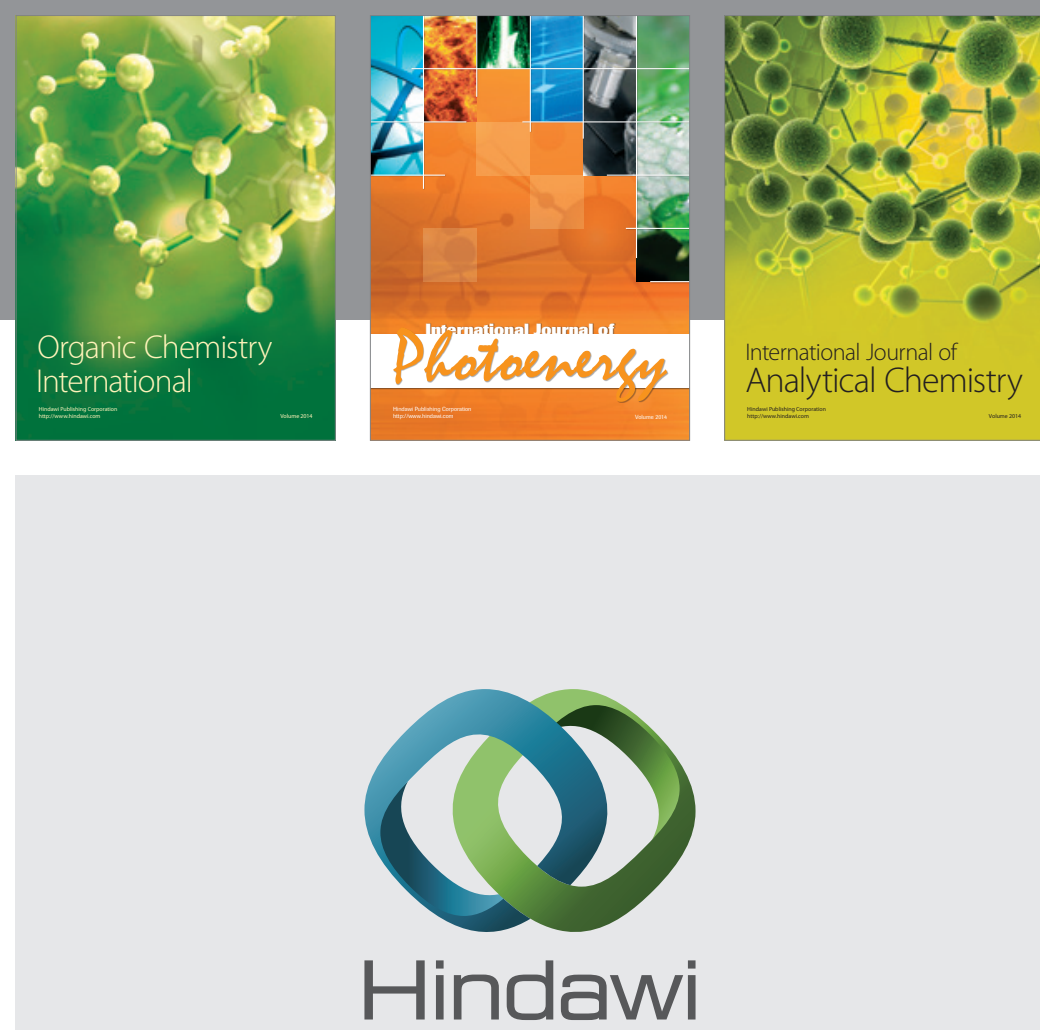

Submit your manuscripts at

http://www.hindawi.com
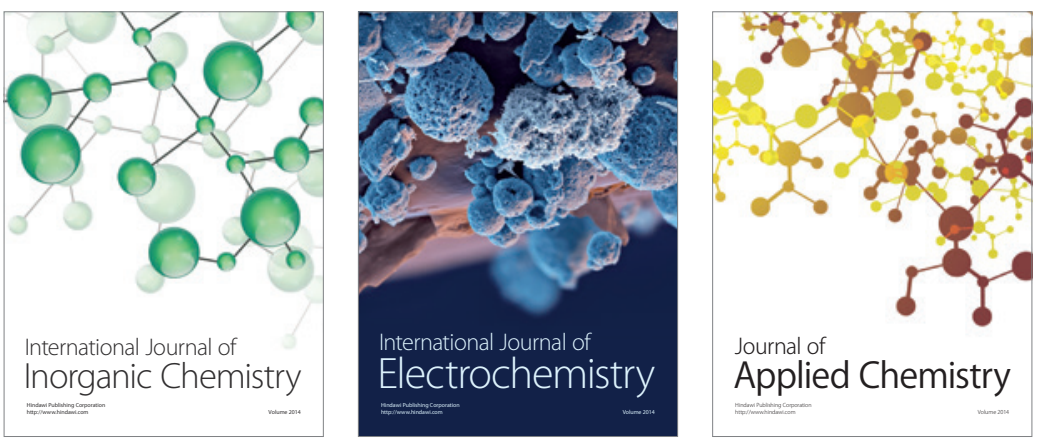

Journal of

Applied Chemistry
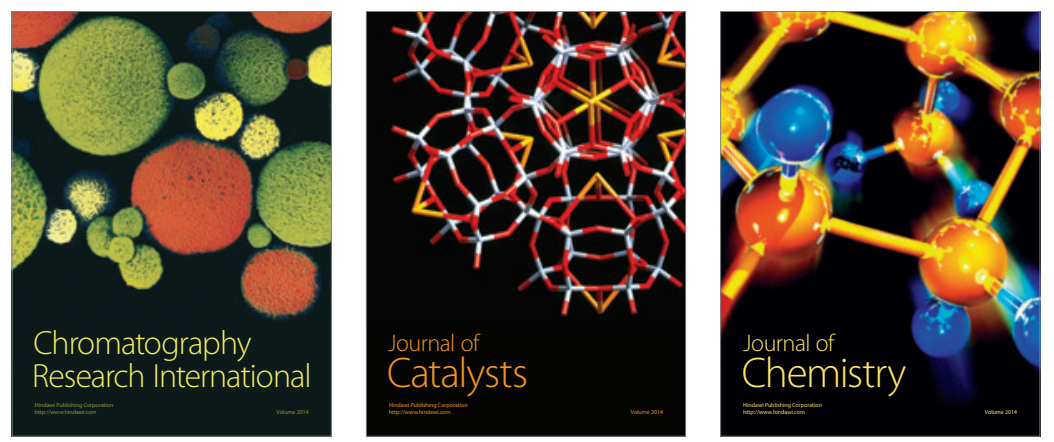
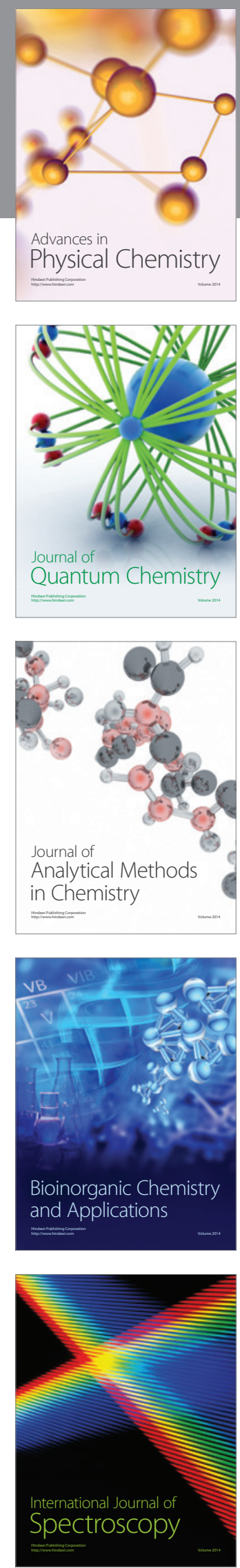